\title{
NOUVELle
}

\section{NOX2, un acteur versatile de la réponse immunitaire, complice de l'infection par le virus de la grippe}

Julie Miesch ${ }^{1}$, Irma Segoviano Vilchis ${ }^{1}$, Sophie Dupré2

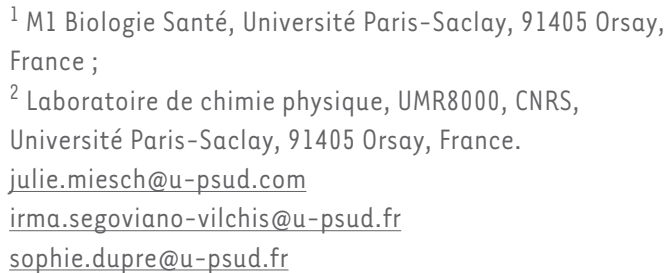

$>$ Avec environ cinq millions de personnes infectées par an dans le monde dont $10 \%$ d'infections mortelles, le virus de la grippe constitue une menace sanitaire et sociale majeure [1]. L'infection par le virus de la grippe chez I'homme s'effectue via le tractus respiratoire. Si le virus réussit à franchir la barrière de mucus, il va alors infecter en premier les cellules épithéliales pulmonaires, puis les macrophages résidents et les cellules dendritiques situées sous l'épithélium [2]. Dans ces cellules, il est reconnu par différents PRR (pattern recognition receptors) et notamment des TLR (Toll-like receptors) localisés au niveau des endosomes (TLR3 et TLR7). Ceux-ci reconnaissent I'ARN viral [3]. Eunice $\varepsilon$. To, et al. - des chercheurs australiens - ont précédemment montré que l'activation des TLR3 et -7 induisait la production de formes réactives de l'oxygène (FRO) par la NADPH oxydase (NOX2) [1]. Cette enzyme, dont la sous-unité catalytique est NOX2, est présente dans les cellules phagocytaires et contribue à la destruction des bactéries et des champignons. Cependant, les effets de la NADPH oxydase restent méconnus dans le cadre d'une infection virale. Plusieurs études suggèrent que les FRO produites par NOX2 favorisent l'infection par le virus de la grippe [4, 5]. Dans un article récent publié dans Nature Communications, les chercheurs australiens montrent que NOX2 est activée lors d'une infection par le virus de la grippe (différents sous-types d'Hemophilus Influenza A) impliquant TLR7, et expliquent comment la production de FRO faciliterait l'infection virale [6].

TLR7 démasque la présence virale et déclenche la production de FRO

Des expériences d'immunofluorescence dans des macrophages alvéolaires primaires murins ont tout d'abord permis aux auteurs de localiser NOX2 et une partie des nucléoprotéines du virus (associées à l'ARN) dans les endosomes précoces. Puis, l'utilisation d'une sonde, qui est internalisée avec les virus et émet une fluorescence lorsqu'elle est oxydée par les $\mathrm{FRO}^{\mathrm{l}}$, a révélé qu'il y avait une production de FRO dans les endosomes. Une telle production a aussi été montrée dans des macrophages alvéolaires humains infectés par différentes souches de virus de la grippe qui ont été, par le passé, responsables de pandémies. L'inhibition des pompes V-ATPases endo/ lysosomales, responsables de l'acidification des endosomes, bloque la production de FRO ; cette dernière n'est cependant pas nécessaire à l'internalisation du virus. Au niveau des endosomes, I'ARN du virus est reconnu par TLR7 [7]. Les auteurs observent effectivement une colocalisation des nucléoprotéines associées à l'ARN viral et de TLR7, ainsi que de TLR7 et NOX2. De plus, la délétion (invalidation génétique) de TLR7, ou celle de la protéine adaptatrice MyD88, abolissent la production des FRO endosomales. Les auteurs ont ensuite cherché

1 II s'agit d'albumine bovine couplée à de l'H2HFF (dihydro-2,4,5,6,7,7'-hexafluorofluorescéine). Cette sonde est internalisée comme les virus et permet donc la détection des FRO dans l'endosome. à identifier quelle protéine, activée via la cascade de signalisation mise en jeu par TLR7, induisait l'activation de la NADPH oxydase. La NADPH oxydase est composée de sous-unités transmembranaires, N0X2 et p22 phox (phagocytic oxidase), de sous-unités cytosoliques ( $p 47^{\text {phox }}$, $\mathrm{p} 40^{\text {phox }}, \mathrm{p} 67^{\text {phox }}$ ) et de la protéine $\mathrm{G}$ Rac (Figure 1). L'assemblage du complexe est nécessaire pour la production de FRO. La phosphorylation de $\mathrm{p} 47^{\text {phox }}$ induit un changement de conformation de celle$\mathrm{ci}$, ce qui permet la translocation du complexe $\mathrm{p} 47^{\text {phox }}-\mathrm{p} 40^{\text {phox }}-\mathrm{p} 67^{\text {phox }}$ à la membrane. Les protéines kinases C (PKC) sont impliquées dans cette phosphorylation [8] et étaient donc de bons candidats. Pour mesurer l'activité de ces kinases, un biosenseur cytoCKAR ${ }^{2}$ [9] a été exprimé dans des macrophages dérivés de la moelle osseuse de souris exposées ultérieurement aux virus. Les auteurs retrouvent bien une augmentation de l'activité de la PKC en présence de virus. Celle-ci ne se produit pas lorsqu'un inhibiteur de l'endocytose est ajouté, ou dans des macrophages de souris dépourvus de TLR7. Cette production endosomale de FRO, dépendante de TLR7, a été également observée pour d'autres virus pénétrant dans la cellule par endocytose.

\footnotetext{
${ }^{2}$ CytoCAR est un bio-senseur constitué des protéines fluorescentes YFP (yellow fluorescent protein) et CFP (cyanin fluorescent protein) et lié à un peptide substrat des PKC. Celui-ci est lui-même relié au domaine FHA2 (forkhead-associated domain) de la protéine de levure rad53p. En absence de phosphorylation du peptide substrat, un transfert d'énergie de fluorescence de type Förster (FRET) a lieu entre les 2 protéines fluorescentes. La phosphorylation du peptide entraine sa liaison au domaine FHA2 via un changement de conformation qui induit une diminution du FRET entre CFP et YFP.
} 


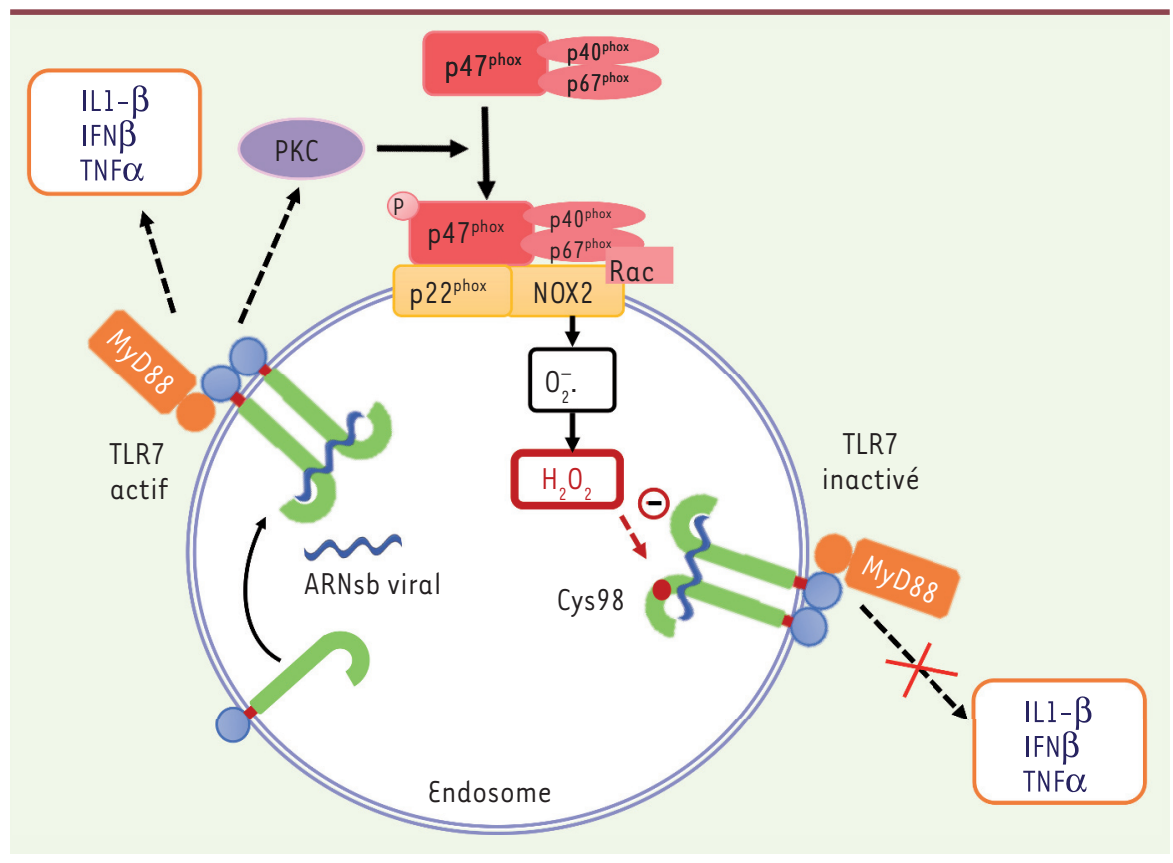

Figure 1. NOX2 inhibe la production de cytokines déclenchée en réponse à une infection par le virus de la grippe. L'ARN simple brin (ARNsb) viral, présent au niveau des endosomes à $\mathrm{pH}$ acide, est reconnu par TLR7. L'activation de la voie de signalisation de TLR7, via l'adaptateur MyD88, induit la production d'interféron $\beta$ (IFN $\beta$ ) et de cytokines pro-inflammatoires: interleukine-6 (IL-6), TNF $\alpha$ (tumor necrosis factor) et IL-1 $\beta$, ainsi que l'activation de la protéine kinase C (PKC). La PKC, à son tour, en phosphorylant notamment $\mathrm{p} 47^{\text {phox }}$ induit l'assemblage et l'activation de la NADPH oxydase NOX2. Les FRO produits par NOX2 vont oxyder la cystéine 98 du TLR7 ce qui inhibe l'activation du récepteur et provoque l'arrêt de la production d'IFN $\beta$ et de cytokines pro-inflammatoires.

\section{Les FRO produites par NOX2 réduisent}

\section{TLR7 au silence}

Eunice $\varepsilon$. To et al. se sont ensuite demandé si les FRO produites par NOX2 influençaient la production de cytokines qui résulte de l'activation des TLR7 par l'ARN viral. L'expression d'interféron $\beta$ (IFN $\beta$ ) et des cytokines pro-inflammatoires IL-6 (interleukine-6), TNF $\alpha$ (tumor necrosis factor) et IL-1 $\beta$ a été analysée par RT-PCR (realtime polymerase chain reaction) dans des macrophages stimulés par l'imiquimod, un agoniste de TLR7. Les auteurs ont ainsi pu observer qu'en présence d'imiquimod et d'apocynine (un inhibiteur de NOX2), l'expression des cytokines est significativement plus importante qu'en présence d'imiquimod seul. Pour vérifier si cette modulation négative des cytokines par les FRO avait lieu in vivo, les chercheurs ont traité des souris sauvages ou déficientes pour NOX2 par de l'imiquimod et ils ont mesuré les ARNm codant les cytokines, $24 \mathrm{~h}$ après ce traitement. L'expression des transcrits codant l'IFN $\beta$ et les cytokines pro-inflammatoires était effectivement significativement plus élevée chez les souris déficientes pour NOX2, confirmant l'effet inhibiteur de cette voie. NOX2 produit de l'anion superoxyde qui peut se transformer (dismutation) en peroxyde d'hydrogène $\left(\mathrm{H}_{2} \mathrm{O}_{2}\right)$. C'est ce dernier qui inhibe l'expression des cytokines via son action sur TLR7. Afin de savoir si certaines cystéines de TLR7, exposées lors de son activation dans l'endosome et cruciales pour la régulation de celle-ci, pouvaient être les cibles $d^{\prime} \mathrm{H}_{2} \mathrm{O}_{2}$, les auteurs ont créé une série d'ADNc codant des récepteurs TLR7 dont une ou plusieurs de ces cystéines étaient mutées. La capacité de ces récepteurs TLR7 mutés à restaurer la production de cytokines dans les macrophages $\mathrm{TLR}^{-/-}$a été analysée en réponse à la stimulation de TLR7. Cette approche de complémentation fonctionnelle a permis d'identifier la cystéine 98 comme une cible potentielle d' $\mathrm{H}_{2} \mathrm{O}_{2}$. La cystéine 98 est un résidu très conservé et spécifique du TLR7. TLR7 est clivé dans l'endosome, les 2 parties seraient ensuite liées par un pont disulfure. Ce clivage conditionne le déclenchement de la signalisation lorsque le récepteur est activé [2], et la cystéine 98 est nécessaire à ce clivage et impliquée dans la formation du pont disulfure [10].

\section{NOX2 facilite l'infection par le virus de} la grippe

Les chercheurs ont ensuite déterminé si ce même mécanisme était opérationnel lors de la stimulation de TLR7 non pas par l'agoniste, mais par le virus de la grippe. L'infection virale par Influenza conduit à l'activation de NOX2 via TLR7. La production de FRO par NOX2 qui en résulte conduit-elle également à diminuer l'expression d'IFN $\beta$ et de cytokines pro-inflammatoires? Celle-ci est effectivement augmentée in vitro dans les macrophages alvéolaires de souris

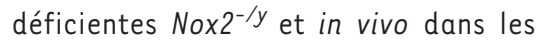

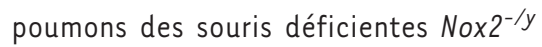
et infectées par Influenza. De plus, chez ces souris, I'IFN $\beta$ est détecté en plus grande quantité dans le fluide bronchoalvéolaire et le sérum, $24 \mathrm{~h}$ après l'infection. D'autres expériences montrent également une augmentation d'anticorps dans le sérum et le fluide bronchoalvéolaire des souris Nox2-/y par rapport aux souris sauvages, 7 jours après l'infection virale. Afin de cibler directement la N0X2 endosomale pour en confirmer son rôle, les auteurs ont utilisé l'inhibiteur NOX2ds-TAT ${ }^{3}$ [11] qu'ils ont lié, via du polyéthylène glycol, à un stéroïde : le cholestanol. Ils montrent que cet inhibiteur se localise dans les endosomes

\footnotetext{
${ }^{3}$ NOX2ds-TAT correspond à la fusion de 9 résidus de la protéine TAT du virus de l'immunodéficience humaine avec 9 résidus de NOX2 qui seraient impliqués dans la liaison à
} p47phox. 
grâce au cholestanol et qu'il diminue in vitro la production de FRO par NOX2. De plus, in vivo, cet inhibiteur, appliqué par voie intra-nasale, réduit fortement I'inflammation au niveau des poumons ainsi que la charge virale.

\section{NOX2 : cible thérapeutique contre le virus de la grippe?}

Dans cet article, Eunice $\varepsilon$. To et al. montrent que la reconnaissance par TLR7 de I'ARN du virus de la grippe entraîne, via la PKC, l'activation de NOX2 et la production de FRO dans les endosomes. Ces FRO inhibent la production de cytokines pro-inflammatoires induite par l'activation initiale de TLR7 par le virus (Figure 1). L'inactivation de TLR7 par les FRO pourrait être un mécanisme protecteur visant à inhiber une réponse inflammatoire contre nos propres ARN ; mécanisme qui permettrait également au virus de la grippe d'augmenter son pouvoir pathogène en retardant la réponse immunitaire. L'inhibition transitoire de NOX2 pourrait donc constituer une approche thérapeutique intéressante pour lutter contre le virus de la grippe. $\diamond$

NOX2, a multifaceted player in the immune response, facilitates influenza virus infection

\section{LIENS D'INTÉRÊT}

Les auteurs déclarent n'avoir aucun lien d'intérêt concernant les données publiées dans cet article.

\section{RÉFÉRENCES}

1. To $\varepsilon \varepsilon$, Broughton BR, Hendricks KS, et al. Influenza $A$ virus and TLR7 activation potentiate NOX2 oxidasedependent ROS production in macrophages. Free Radic Res 2014 ; 48 : 940-7.

2. Descamps D, Manoury B. Détection du virus de l'influenza par les récepteurs Toll-like des interactions majeures dans le contrôle des réponses antigrippales. Virologie $2013 ; 17: 369-78$

3. Iwasaki A, Pillai PS. Innate immunity to influenza virus infection. Nat Rev Immunol 2014 ; 14 : 315-28.

4. Snelgrove RJ, Edwards L, Rae AJ, et al. An absence of reactive oxygen species improves the resolution of lung influenza infection. Eur J Immunol 2006 ; $36: 1364-73$.

5. Vlahos R, Stambas J, Broughton BRS, et al. Inhibition of Nox2 oxidase activity ameliorates Influenza A virus-induced lung inflammation. Plos Pathog 2011 ; 7: el001271.

6. To $\varepsilon$, Vlahos $R$, Luong $R$, et al. Endosomal NOX2 oxidase exacerbates virus pathogenicity and is a target for antiviral therapy. Nat Commun 2017 ; 8:69.

7. Diebold SS, Kaisho T, Hemmi H, et al. Innate antiviral responses by means of TLR7-mediated recognition of single-stranded RNA. Science 2004 ; 303 : 1529-31.

8. Paula Nunes P, Demaurex N, Mary C. Dinauer MC. Regulation of the NADPH Oxidase and Associated Ion Fluxes During Phagocytosis. Traffic 2013 ; 4 : 1118-31.

9. Violin JD, Zhang J, Roger Y, Tsien Ry, et al. A genetically encoded fluorescent reporter reveals oscillatory phosphorylation by protein kinase C. J Cell Biol 2003 ; $161: 899-909$.

10. Kanno A, Yamamoto C, Onji M, et al. Essential role for Toll-like receptor 7 (TLR7)-unique cysteines in an intramolecular disulfide bond, proteolytic cleavage and RNA sensing, Int Immunol $2013 ; 25: 413-22$.

11. Rey FE, Cifuentes ME, Kiarash A, et al. Novel competitive inhibitor of NAD (P)H oxidase assembly attenuates vascular $\mathrm{O}_{2}^{-}$and systolic blood pressure in mice. Circ Res $2001 ; 89: 408-14$.

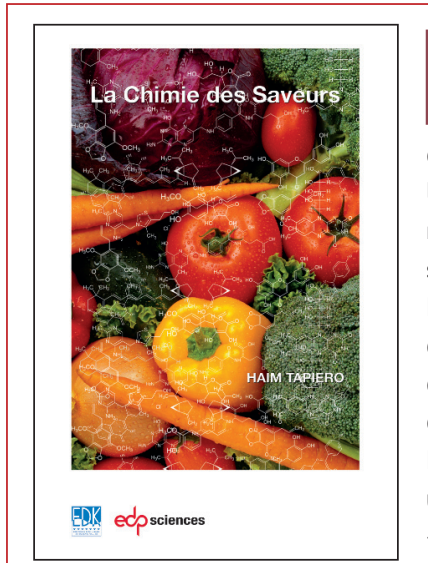

ISBN : 978-2-7598-1137-3 180 pages a cuisine est une science. II existe une relation étroite entre élaborer une recette et entreprendre une recherche scientifique. Quelle que soit l'origine d'une recette, d'un livre ou inventée, il faudra faire le choix des ingrédients, les mélanger et les cuire de manière appropriée afin de ne pas altérer les substances actives qui composent les ingrédients.

Une fois la cuisson terminée, il faudra analyser le goût et si nécessaire prévoir son amélioration. Améliorer une recette nécessite de connaître le ou les processus qui interviennent dans le développement des arômes, des saveurs et de la texture. Cette approche est similaire à celle développée par le scientifique.

La relation entre l'élaboration des recettes, les substances nutritives qui composent les ingrédients et la santé de l'homme est issue de plusieurs disciplines de la recherche fondamentale et clinique. Au cours des dernières années, de nombreux travaux scientifiques ont été publiés sur le rôle de la nutrition et la réduction des risques dans les pathologies comme les maladies cardio-vasculaires ou les cancers.

Le but principal de cet ouvrage a été d'identifier la structure chimique des composants actifs des ingrédients utilisés en cuisine (légumes, herbes aromatiques, épices) et qui entrent dans la préparation des recettes pour « végétariens » et « omnivores».

À retourner à EDP Sciences, 17 avenue de Hoggar, 91944 Les Ulis Cedex A - Tél. : 0149856069 - Fax : 0149850345 - E-mail : edk@edk.fr NOM : Prénom :

Adresse :

Code postal :

Prénom:

Pays :

Fonction :

Je souhaite recevoir l'ouvrage La chimie des Saveurs : $20 €+3 €$ de port $=\mathbf{2 3} €$ TTC

en ................ exemplaire, soit un total de

$€$

$\square$ Par chèque, à l'ordre de $\mathbf{E} \mathbf{D} \mathbf{K}$

Par carte bancaire :

$\square$ Visa $\square$ Eurocard/Mastercard

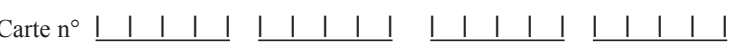

Signature :

Date d'expiration : $\quad 1 \quad 1 \quad 1 \quad 1$

$\mathrm{N}^{\circ}$ de contrôle au dos de la carte : 